\title{
Surface-Enhanced Raman Scattering
}

\author{
Mustafa Culha, ${ }^{1}$ Nickolay Lavrik, ${ }^{2}$ Brian M. Cullum, ${ }^{3}$ and Simion Astilean ${ }^{4}$ \\ ${ }^{1}$ Department of Genetics and Bioengineering, Yeditepe University, Atasehir, Kadıkoy, 34755 Istanbul, Turkey \\ ${ }^{2}$ Center for Nanophase Materials Sciences, Oak Ridge National Laboratory, Oak Ridge, TN 37830, USA \\ ${ }^{3}$ Department of Chemistry and Biochemistry, University of Maryland Baltimore County, 1000 Hilltop Circle, Baltimore, \\ MD 21250, USA \\ ${ }^{4}$ Nanobiophotonics Center, Institute for Interdisciplinary Experimental Research in Nanobioscience, Faculty of Physics, \\ Babes-Bolyai University, T. Laurian 42, 400271 Cluj-Napoca, Romania
}

Correspondence should be addressed to Mustafa Çulha, mculha@yeditepe.edu.tr

Received 10 June 2012; Accepted 10 June 2012

Copyright (C) 2012 Mustafa Çulha et al. This is an open access article distributed under the Creative Commons Attribution License, which permits unrestricted use, distribution, and reproduction in any medium, provided the original work is properly cited.

Surface-enhanced Raman scattering (SERS) is welcomed by researchers in a wide range of fields from physics to medicine. The reason behind this broad interest in SERS is a number of its unique features, such as high sensitivity, capability to provide chemically specific information about components of a sample, and applicability to a range of biological and nonbiological samples. The most important factor in its exponential growth is the interdisciplinary nature of the technique. Indeed, this issue covers a number of diverse and promising applications of SERS and further demonstrates its interdisciplinary landscape.

K. D. Alexander et al. investigated the relationship between length and SERS signal strength in gold nanoparticle (AuNP) chains. The spherical AuNPs with $60 \mathrm{~nm}$ sizes were brought into 1-9 nanoparticle chains in the channels $\left(80 \mathrm{~nm}\right.$ in width and $70 \mathrm{~nm}$ in depth) patterned on an $\mathrm{SiO}_{2}$ substrate by applying capillary force deposition. The SERS enhancement factor strength for the chains was compared to the numerical predictions. It was found that while dimer nanoparticles offered large enhancement over single particles, increasing the chain length more than two nanoparticles does not appreciably improve the SERS enhancement. It was also stated that the surface morphology could be an important contributing factor that cannot be controlled with the current technology.

A. Polemi et al. reported "Sensing properties of a FabryPerot dielectric structure and dimer nanoparticles." In their study, the Fabry-Perot structures, disc dimers, and bowtie elements, are prepared by placing a superstrate with a high permittivity on a substrate with a very low permittivity to establish the resonance condition. They demonstrated that the array of disc dimers and bowties could effectively increase the SERS gain. They conclude their report by suggesting that other nanoparticle geometries and new array arrangements could be used for sensing.

There is a strong interest to employ the technique for microbial detection and identification. However, there are still a number of problems that need to be addressed before SERS can be efficiently used for identification of real microbial and cellular samples. One of the most important issues in its microbial applications is the reproducibility. M. Çulha et al. describes a novel substrate constructed from silver nanoparticles (AgNPs) to obtain more reproducible SERS spectra for bacterial identification. They compared the results of the study to their previously reported results obtained from the samples prepared with simple mixing and convective assembly. They concluded that the prepared SERS substrate generates similar reproducibility to the convective assembly but more improved reproducibility over simple mixing.

M. R. Goncalves et al. reported the investigation of hotspot formations on triangular silver nanostructures fabricated by thermal evaporation by examining the SERS of thiols and dye molecules adsorbed on these nanostructures. They observed that the hotspots were localized at the edges and corners of the silver triangular particles. They found that the strong and fast fluctuating SERS activity on the fabricated structures is due to the contamination of the nanostructure with amorphous carbon during the thermal evaporation process. They also observed that the adsorption of thiols and dye molecules on the newly prepared surfaces could reduce the undesired SERS activity. 
S. Boca et al. reported a strategy to prepare small aggregates of dimers or trimers of AuNPs by capturing from solution and interlocking them into a polymeric cage of thiol-modified poly(ethylene) glycol (PEG-SH). Then, these small aggregates are used as sensing units by transferring into living cells as SERS tags. They demonstrated the usefulness of the approach by using human retinal cells.

O. Rabin and S. Lee reported the variations in SERS enhancement in clusters of assembled silver nanocubes with different sizes and configurations, and laser frequency and polarization. The assembly of silver nanocubes into small clusters on patterned silicon substrate by either vertical deposition or electrophoretic deposition was achieved. The study concluded that the face-to-face linear configuration of the nanocube clusters was not effective for the improvement in SERS enhancement as compared to the linear clusters where nanocubes are located along to an edge.

C. K. Klutse et al. reviewed "Applications of selfassembled monolayers (SAMs) in SERS." SAMs can enhance analytical capacity of SERS substrates improving their longterm stability, selectivity, and reproducibility. The authors focus on the use of SAMs to improve SERS enhancement further. They demonstrate the applicability of the approach from the novel multilayered SERS substrates developed in their laboratory. They claim that SERS enhancement could be greater than 20 -fold compared to conventional single layer SERS substrates.

In this special issue, the editors also reviewed several promising but less frequently highlighted trends in preparation of SERS active substrates as well as some biological applications of SERS. While there is a consensus that SERS substrates fabricated using deterministic nanoscale patterning and wafer-level processing are very promising, broader analytical applications of such substrates are lagging behind compared to SERS-active systems prepared according to more conventional colloidal synthesis and assembly routes. This trend is particularly strong in biological applications of SERS, including detection and identification of proteins, microorganisms, and cellular samples; selected recent examples of which are highlighted in the review.

Mustafa Culha

Nickolay Lavrik

Brian M. Cullum

Simion Astilean 

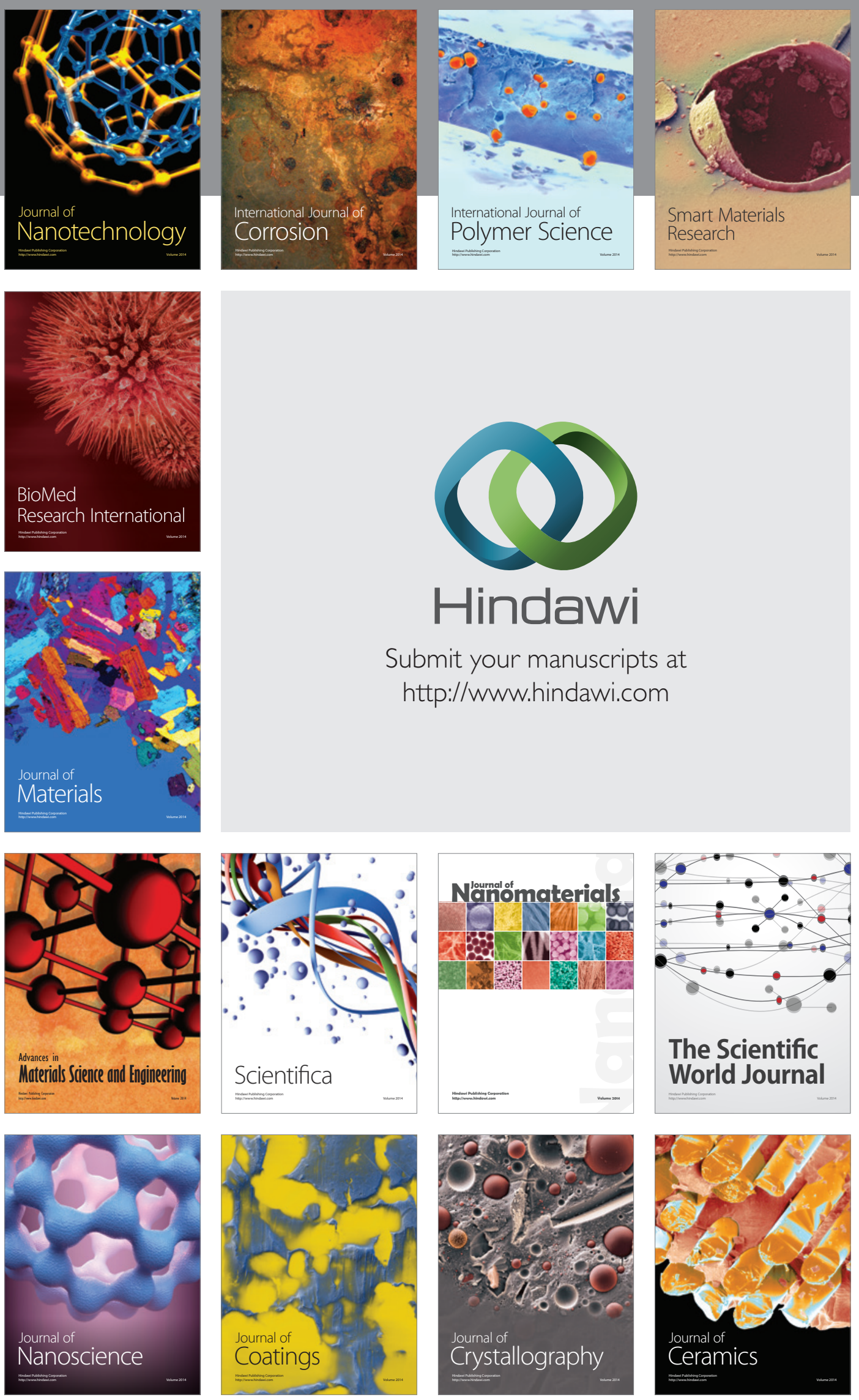

The Scientific World Journal

Submit your manuscripts at

http://www.hindawi.com

\section{World Journal}

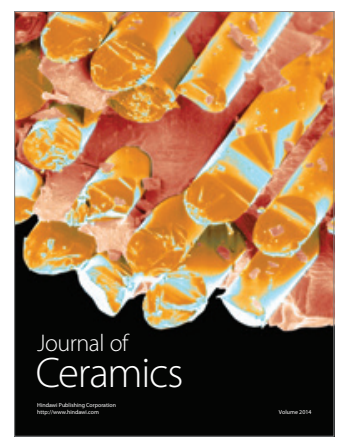

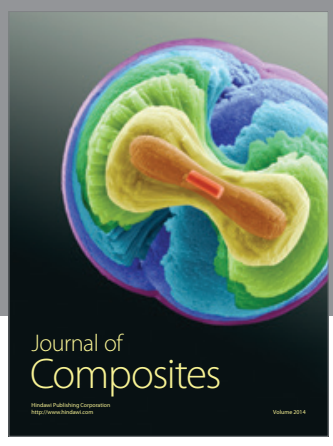
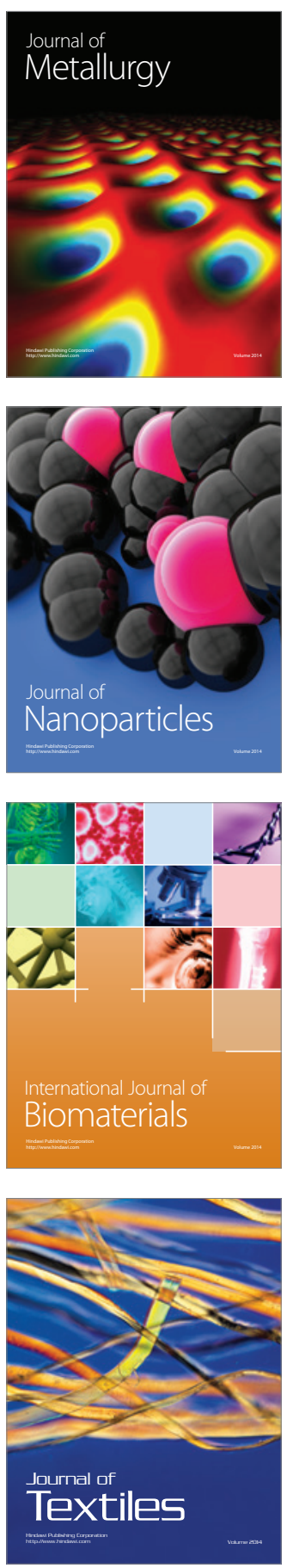